\section{Platelet Membrane Glycoprofiling in a PMM2-CDG Patient}

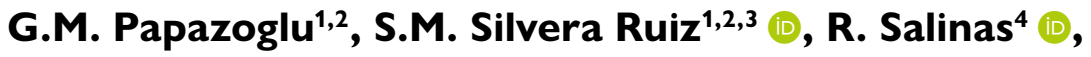 \\ M.I. Pereira ${ }^{5}$, M.A. Cubilla ${ }^{1,2}$, F. Pesaola ${ }^{2,6} \oplus$, S. Ghione \\ N. Ramadán ${ }^{8}$, I. Martinez-Duncker ${ }^{4}$, C.G. Asteggiano, ${ }^{1,2,9}$ (1)
}

Journal of Inborn Errors of Metabolism \& Screening 2021, Volume 9: e20200030 DOI: https://doi.org/10.1590/2326-4594 JIEMS-2020-0030

\begin{abstract}
Congenital disorders of glycosylation (CDG) are metabolic hereditary diseases caused by defects in the synthesis of glycoconjugates. CDG have been described in sugar-nucleotide biosynthesis and transporter, glycosyltransferases, vesicular transport, as well as in lipid biosynthesis and glycosylphosphatidylinositol anchors. PMM2-CDG is caused by mutations in the phosphomannomutase-2 (PMM2) gene and shows autosomal recessive inheritance. It affects all organs and tissues, ranging from severe psychomotor retardation to moderate intellectual disability. Alterations in the primary haemostatic system have been reported in these patients and they can lead to severe bleeding or excessive thrombosis with subsequent vascular insufficiency. Despite of being the most common CDG, platelet glycosylation and sialylation defects in PMM2-CDG patients remain incompletely characterized. In this study, we applied a lectin-based flow cytometry approach to report the first characterization of the highly glycosylated platelet membrane glycan profile in a PMM2-CDG patient. In the PMM2-CDG patient's platelet samples, a decreased binding of SNA lectin, indicative of reduced terminal a-2-6 sialic acid content, and an increased binding of PNA lectin, suggesting desialylation of $\beta-1-N-$ acetylgalactosamine residues, were observed. Reduced expression of terminal sialic acids in platelet membrane glycoproteins may contribute to the increased risk of hemorrhage reported in these patients by promoting platelet clearance and thrombocytopenia.
\end{abstract}

Keywords: mass spectrometry, platelet membrane, glycoprofile, PMM2-CDG, congenital disorders of glycosylation.

\section{Introduction}

Congenital disorders of glycosylation (CDG) encompass a group of inborn errors of metabolism involving more than 130 genes influencing different glycosylation pathways [1,2]. Alterations in $\mathrm{N}$-linked, $\mathrm{O}$-linked, and combined $\mathrm{N}$-O-linked glycosylation, as well as lipid glycosylation defects, are distinctly associated with the various CDG [3]. PMM2-CDG (formerly known as CDG-Ia) is the first clinically described and most common type of CDG [4]. PMM2-CDG is caused by mutations in the PMM2 gene [OMIM *601785], which encodes phosphomannomutase 2 (EC 5.4.2.8), an enzyme necessary for the synthesis of GDP-mannose [4]. PMM2-CDG is an autosomal recessive, pan-ethnic disorder with an estimated incidence of 1/20,000 live birth [5]. Along with the typical neurological and multisystemic clinical presentation, these patients often present severe and frequently lethal thrombohemorrhagic complications (bleeding or thrombosis), due to platelet alterations associated with a decreased response to agonists [4,6-9]. Platelet surface membrane glycoproteins (GPIb/IX/V, GPIIb/IIIa) are fundamental in the hemostatic process. However, little is known about the consequences of hypoglycosylation in the platelets of PMM2-CDG patients. De la

\footnotetext{
'Universidad Nacional de Córdoba, Facultad de Ciencias Médicas, Hospital de Niños de la Sma. Trinidad, Centro de Estudio de las Metabolopatías Congénitas, Córdoba, Argentina.

${ }^{2}$ Consejo Nacional de Investigaciones Científicas y Técnicas, Buenos Aires, Argentina.

${ }^{3}$ Universidad Nacional de Córdoba, Facultad de Ciencias Químicas, Centro de Investigaciones en Bioquímica Clínica e Inmunología, Córdoba, Argentina.

${ }^{4}$ Universidad Autónoma del Estado de Morelos, Instituto de Investigación en Ciencias Básicas y Aplicadas, Centro de Investigación de Dinámica Celular, Laboratorio de Glicobiología Humana y Diagnóstico Molecular, Cuernavaca, México.

${ }^{5}$ Laboratorio de Inmunología, Hospital de Niños de la Sma. Trinidad, Córdoba, Argentina.

${ }^{6}$ Hospital de Niños de la Sma. Trinidad, Programa de Investigación Traslacional de Lipofuscinosis Ceroidea Neuronal, Córdoba, Argentina.

${ }^{7}$ Argenlab San Francisco, Córdoba, Argentina.

${ }^{8}$ Fundación para el Progreso de la Medicina, Córdoba, Argentina.

'Universidad Católica de Córdoba, Facultad de Ciencias de la Salud, Carrera Medicina, Córdoba, Argentina.
}

Received December 28, 2020. and in revised form July 04, 2021 Accepted for publication July 09, 2021.

\section{Corresponding Author:}

Carla Gabriela Asteggiano, Email: asteggianocarla@hotmail.com 
Morena et al. evaluated RCA120 lectin binding to PMM2-CDG platelets and the expression of platelet membrane $N$-glycoproteins (GPIba and GPIIIa) by flow cytometry. In their study, increased binding of RCA120, which recognizes terminal $\beta$-galactose residues, suggested decreased surface sialic acid levels in PMM2CDG platelets [9].

Sialic acids are hydrophilic, electronegatively charged monosaccharides with a common nine-carbon backbone that are usually incorporated at terminal glycan positions during glycan remodeling events. Due to their location and ubiquitous distribution, sialic acids can mediate or modulate a wide variety of physiological and pathological processes [10]. It is therefore attractive to hypothesize that increased platelet reactivity in PMM2-CDG patients may be caused by a deficient glycosylation of platelet membrane $\mathrm{N}$-glycoproteins. To evaluate this hypothesis, we conducted a flow cytometry experiment using a panel of 7 glycoform-specific lectins to provide the first description of the glycan profile of the platelet surface membrane in a PMM2-CDG patient.

We used AAL, RCA-I, SNA, UEA-I, CON-A, PNA, and MAA-I, which binds to different glycans as shown in Table 1.

\section{Methods}

\section{Subjects}

The PMM2-CDG patient was referred by pediatricians and diagnosed at the Center for the Study of Congenital Metabolopathies (CEMECO), Children's Hospital, in Córdoba, Argentina [6]. The control group consisted of six healthy volunteers (25-55 yearsold women) that complied with the following inclusion criteria: normal platelet count $(150-450 \mathrm{pq} / \mu \mathrm{L})$ and not presenting any chronic pathology nor haemostatic alterations. According to the 2005 UNESCO Universal Declaration on Bioethics and
Human Rights (https://en.unesco.org/themes/ethics-science-andtechnology/bioethics-and-human-rights), participants were duly informed of the aim and procedures of this study and provided with a written informed consent prior to blood extraction. This study was approved by the institutional review boards of CIEISEthics Committee, Children’s Hospital, Córdoba, Argentina.

\section{Analysis of haemostatic parameters and platelet function}

Blood samples were collected from the PMM2-CDG patient, and platelet-rich plasma (PRP) and platelet-poor plasma (PPP) were obtained as previously described [11]. The optical density of the PRP preparation was measured at $37^{\circ} \mathrm{C}$ in a Crono-Log aggregometer (Crono-Log Corp., PA, USA). PPP (clear plasma) and PRP (cloudy or opaque plasma) were used to calibrate the instrument to define maximum (100\%) and baseline (0\%) light transmission (aggregation), respectively $[11,12] 1$.

In vitro aggregation tests were conducted in the presence of different agonists: ADP $(2.5 \mu \mathrm{M}$ and $5 \mu \mathrm{M})$, epinephrine $(5 \mu \mathrm{M})$, arachidonic acid $(1 \mathrm{mM})$, collagen $(8 \mu \mathrm{g} / \mathrm{mL})$, and ristocetin $(1.5 \mathrm{mg} / \mathrm{mL})$. The PRP of a healthy individual, without prior history of bleeding or thrombosis, was used as control in each determination.

Plasma levels of coagulation factors and inhibitors were also determined. These included Protein S (coagulometric method), Protein C (chromogenic method), Antithrombin III, and coagulation factors III, V, VII, VIII, IX, X, and XI [13,14]. In addition, prothrombotic factors, such as lupus anticoagulant and anticardiolipin, were also measured. All these determinations were made using commercial kits, following the recommendations of the International Society of Thrombosis and Haemostasis (ISTH). Finally, a complete cytological analysis of peripheral blood was carried out to evaluate blood's formed elements (i.e., leukocyte, erythrocyte, and platelet counts) (Table 2).

Table 1. Panel of Lectins used and glycans specifity.

\begin{tabular}{lcc}
\hline Lectin & Sugar Specifity & Concentration ( $\boldsymbol{H g}_{\mathbf{m}} \mathbf{m}$ ) \\
\hline Aleurian Aurantia (AAL) & Fucose-a 1,6 & 0,01 \\
Ricinus Communis Agglutinin I (RCA-I) & -Galactose & 0,02 \\
Sambucus Nigra (SNA) & Sialic acid -2,6 & 0,01 \\
Concanavalina A (CON-A) & a-mannose & 0,01 \\
Ulex Europaeus Agglutinin I (UEA-I) & a-Fucose & 0,01 \\
Peanut Agglutinin (PNA) & B-1, N-acetylgalactosamine & 0,1 \\
Maackia Amurensis (MAA-I) & (Core 1 O-Glycans) & 0,02 \\
\hline
\end{tabular}


Table 2. Haemostatic's determinations in PMM2-CDG Patient.

\begin{tabular}{|c|c|c|}
\hline Haemostatic studies & ARG02 & Normal Value \\
\hline KPTT & $42 "$ & $30 "-43 "$ \\
\hline Quick & $80 \%$ & $70 \%-120 \%$ \\
\hline Prothrombin time & $16 "$ & $14 "-18 "$ \\
\hline Fibrinógen & $288 \mathrm{mg} / \mathrm{dl}$ & $150-350 \mathrm{mg} / \mathrm{dl}$ \\
\hline \multicolumn{3}{|l|}{ Coagulation factors } \\
\hline Factor II & $143 \%$ & $70 \%-120 \%$ \\
\hline Factor $\mathrm{V}$ & $93 \%$ & $70 \%-120 \%$ \\
\hline Factor VII & $128 \%$ & $70 \%-120 \%$ \\
\hline Factor $X$ & $81 \%$ & $70 \%-120 \%$ \\
\hline Factor VIII & $100 \%$ & $50 \%-150 \%$ \\
\hline Factor IX & $53 \%$ & $70 \%-120 \%$ \\
\hline Factor XI & $65 \%$ & $70 \%-120 \%$ \\
\hline \multicolumn{3}{|l|}{ Coagulation inhibitors } \\
\hline Protein S & $140 \%$ & $70 \%-120 \%$ \\
\hline Protein C & $86 \%$ & $75 \%-140 \%$ \\
\hline Antithrombin III & $89 \%$ & $80 \%-120 \%$ \\
\hline \multicolumn{3}{|l|}{ Prothrombotic factors } \\
\hline Lupus Anticoagulant & NEGATIVE & \\
\hline Anticardiolipins & NEGATIVE & \\
\hline Beta 2 Glycoprotein I & NEGATIVE & \\
\hline
\end{tabular}

\section{Platelet isolation}

PRP was obtained through platelet fractionation and processing of $8 \mathrm{~mL}$ of whole blood in a tube containing $3.8 \%(0.109 \mathrm{M})$ sodium citrate as anticoagulant (dilution 1:10). The cell suspension was centrifuged $15 \mathrm{~min}$ at low revolutions $(200 \mathrm{xg}$ ) to obtain PRP containing approximately $70 \%$ of the platelets present in the original whole blood sample. Then, paraformaldehyde ( $1 \%$ final concentration) was added to fix the platelets and prevent their activation in the next centrifugation step (10 min, $900 \mathrm{xg}$ ). Finally, the PRP pellet was suspended in phosphate buffer saline (PBS) at a final platelet concentration of $2 \times 10^{5} \mathrm{cells} / \mathrm{mm}^{3}$.

\section{Analysis of platelet membrane glycan profile}

Glycan profiling of platelet surface membranes was performed in paraformaldehyde-fixed, PBS washed platelet aliquots $(50 \mu \mathrm{L}$; $2 \times 10^{5} \mathrm{cells} / \mathrm{mm}^{3}$ ) from a PMM2-CDG patient and six controls. The platelet population was identified with an anti-CD41-PE antibody (BD Biosciences, USA). Each determination was made in triplicate. Dual CD41/lectin staining was developed by incubating PRP samples with the anti-CD41 antibody for $30 \mathrm{~min}$, followed by an incubation for $20 \mathrm{~min}$ at room temperature (RT) with biotinylated lectins [AAL, RCA-I, SNA, UEA-I, CON-A, PNA, and MAA-I (Vector Laboratories, USA)] that bind to different glycans (Table 1) [15]. The samples were then incubated with streptavidin-Alexa Fluor-647 (1 $\mu \mathrm{g} / \mu \mathrm{L}$, Vector Laboratories) for $20 \mathrm{~min}$ at RT and washed twice with PBS. Glycanprofiles of platelet membranes were evaluated by flow cytometry in a BD FACS Canto IIFlow Cytometer and FACS DIVA software (Becton Dickinson, USA). For each lectin assayed, the corresponding mean fluorescence intensity (MFI) was compared between the PMM2-CDG patient's samples and those of controls. Flow cytometry data were analyzed with the FlowJo v10 program.

\section{Statistical Analysis}

Since our research included only one study subject, standard statistical analyses to compare mean differences between groups could not be performed. To overcome this issue, the patient's lectin fluorescence values were analyzed applying the statistical method suggested by Mycroft et al. (2002) [16]. Briefly, we calculated the minimum F-values with $\mathrm{p}<0.05$ (updated F-values) by simulating 100,000 random measurement outcomes considering different numbers of controls and ratios of patient-to-control variance. Then, after applying a standard repeated measures design (using all the patient's replica values), the resulting F-value was compared to the updated F-values to determine the significance level of the differences observed between the patient and the controls. All the patient's lectin fluorescence values were analyzed as described above [17]. GraphPadv6.01 (GraphPad Software, La Jolla, California, USA) was used for statistical analysis. Results were expressed as mean \pm standard error of the mean (SEM) and $\mathrm{p}<0.05$ was considered as significant. 


\section{Results}

\section{Clinical data and biochemical analyses}

The PMM2-CDG patient is a ten-year-old boy with developmental disability, hypotonia, hyporeflexia, strabismus with optic nerve atrophy and macular hypoplasia, macrocephaly, inverted nipples, and increased gluteal fat pads. Serum transferrin isoelectro focusing revealed hypoglycosylated forms of the plasma protein transferrin, synthesized in the liver, denoting a CDG type I profile. Since DNA variants in the PMM2 gene are the most frequent cause of this hypoglycosylation profile, and considering the patient's clinical phenotype, the PMM2 gene was sequenced. As already reported by our group, two missense variants, c.422G $>$ A (p.Arg141His) and c.415G $>$ A (p.Glu139Lys), were found in exon 5 of the PMM2 gene [6]. The patient had no history of haemostatic and/or platelet alterations, except for mild pericardial effusion detected in 2016 after a lung infection that was monitored periodically.

After collecting a fresh blood sample from the PMM2-CDG patient, we conducted plasma transferrin isoelectric focusing (TfIEF) analysis that revealed hypoglycosylated transferrin forms consistent with a CDG type I profile (Figure 1). Peripheral blood cytology showed normal morphology and counts for red and white blood cells, as well as a normal platelet count $\left(381 \times 10^{3} / \mathrm{mm}^{3}\right.$; NV: $\left.150-450 \times 10^{3} / \mathrm{mm}^{3}\right)$. As shown in Table 2, prothrombin, quick fibrinogens, and coagulation inhibitor levels in the patient were in the normal range, whereas KPTT values were at the normal range's upper limit. The activities of coagulation factors II and VII were above normal standards, while those of Factor IX and XI were below normal range. Antithrombin III and Protein C values were normal, whereas Protein $S$ activity was above range. In turn, negative antibody test results were obtained for the prothrombotic factors lupus anticoagulant and anticardiolipin.

\section{Platelet Function Studies}

The platelet aggregation status of the patient was determined in a real-time aggregometer using PRP samples [12] after independent addition of ADP, arachidonic acid, collagen, and ristocetin. Platelet aggregation is typically observed as a curve in a platelet aggregation (\%) vs. time graph. The process is typically biphasic (primary and secondary aggregation) with 5 different stages: 1. An initial increase in light transmission attributable to PRP dilution by the agonist; 2 . A slight decrease in transmission as platelets change shape; 3 . An acute increase in transmission as platelets aggregate (primary aggregation); 4. A brief plateau or

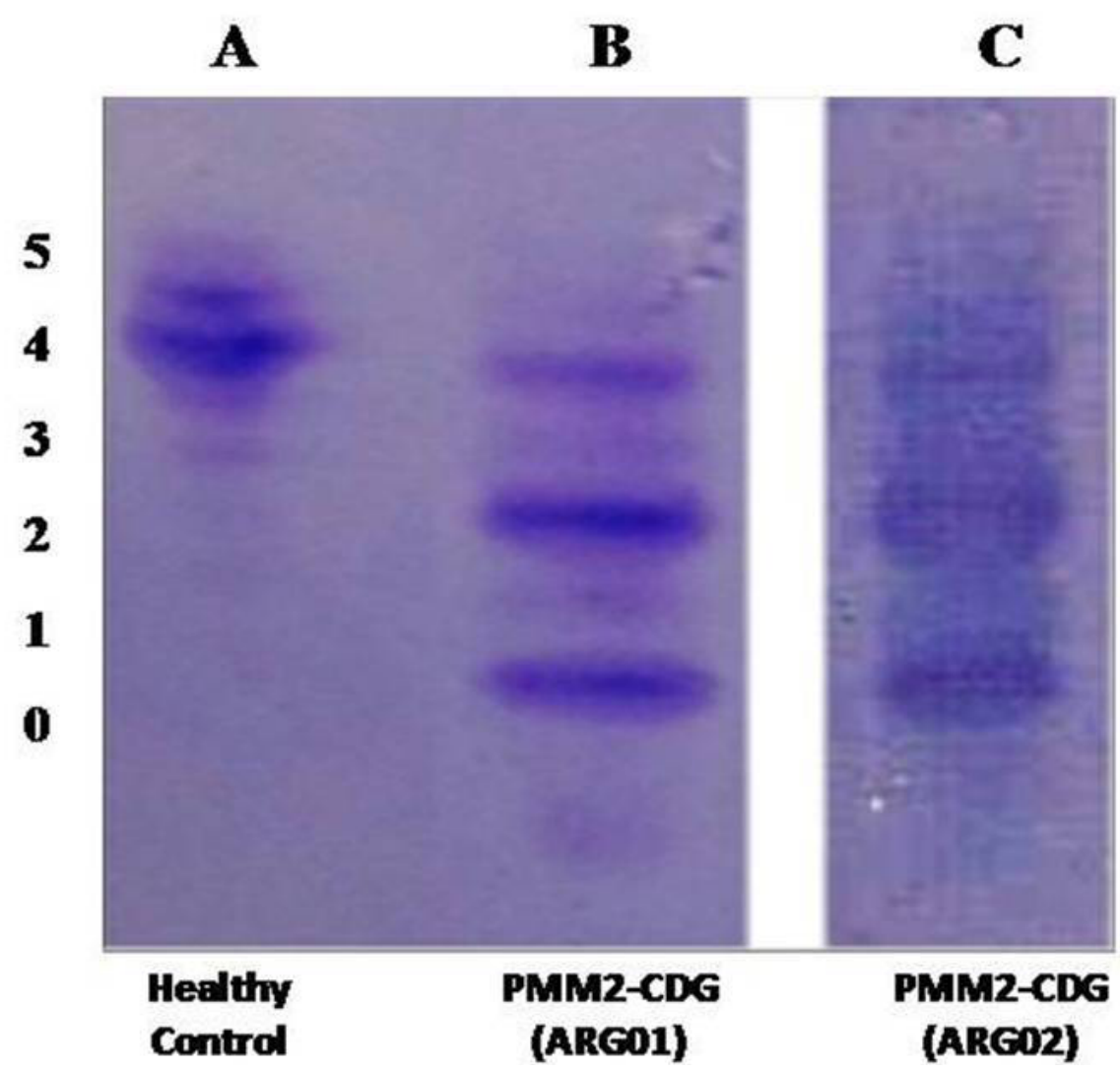

Figure 1. Plasma transferrin isoelectric focusing (TfIEF) analysis.

The number of terminal sialic acid residues in plasma transferrin (0-5) is indicated. Line $\mathbf{A}$ corresponds to a healthy control subject with a normal TfIEF pattern. Lines $\mathbf{B}$ and $\mathbf{C}$ correspond to samples from two patients (AR01 and AR02) with a CDG type I pattern consistent with PMM2-CDG. 
decrease in aggregation; 5 . Secondary aggregation stimulated by the secretion of platelet granules [11,12]. In the PMM2-CDG patient, decreased primary aggregation and absent secondary aggregation were observed in response to both ADP $(2.5 \mu \mathrm{M}$ and $5 \mu \mathrm{M})$ and epinephrine $(5 \mu \mathrm{M})$. In contrast, aggregation was normal upon addition of collagen $(1 \mu \mathrm{g} / \mathrm{mL})$, arachidonic acid $(1 \mathrm{mM})$, or ristocentin $(1.5 \mathrm{mg} / \mathrm{mL})$.

\section{Platelet glycan profiling}

No significant differences in CD41-positive cells were observed between the PMM2-CDG patient (Figure 2A and B) and healthy controls (Figure 2C and D). A panel of 7 biotinylated lectins labeled with streptavidin Alexa Fluor ${ }^{\circ}-647$ antibody was assessed (Table 1) to obtain the platelet membrane glycoprofile by flow cytometry (Table 2).

As shown in Figure 3, no significant differences in platelet binding to AAL, CON-A, UEA-I, RCA-I, and MAA-I were observed between patient and control samples. In contrast, compared to control specimens, significantly higher and lower mean fluorescence intensity MFI values for PNA (Figure 4) and SNA (Figure 5) lectins, respectively, were observed in the patient's samples.

\section{Patient}
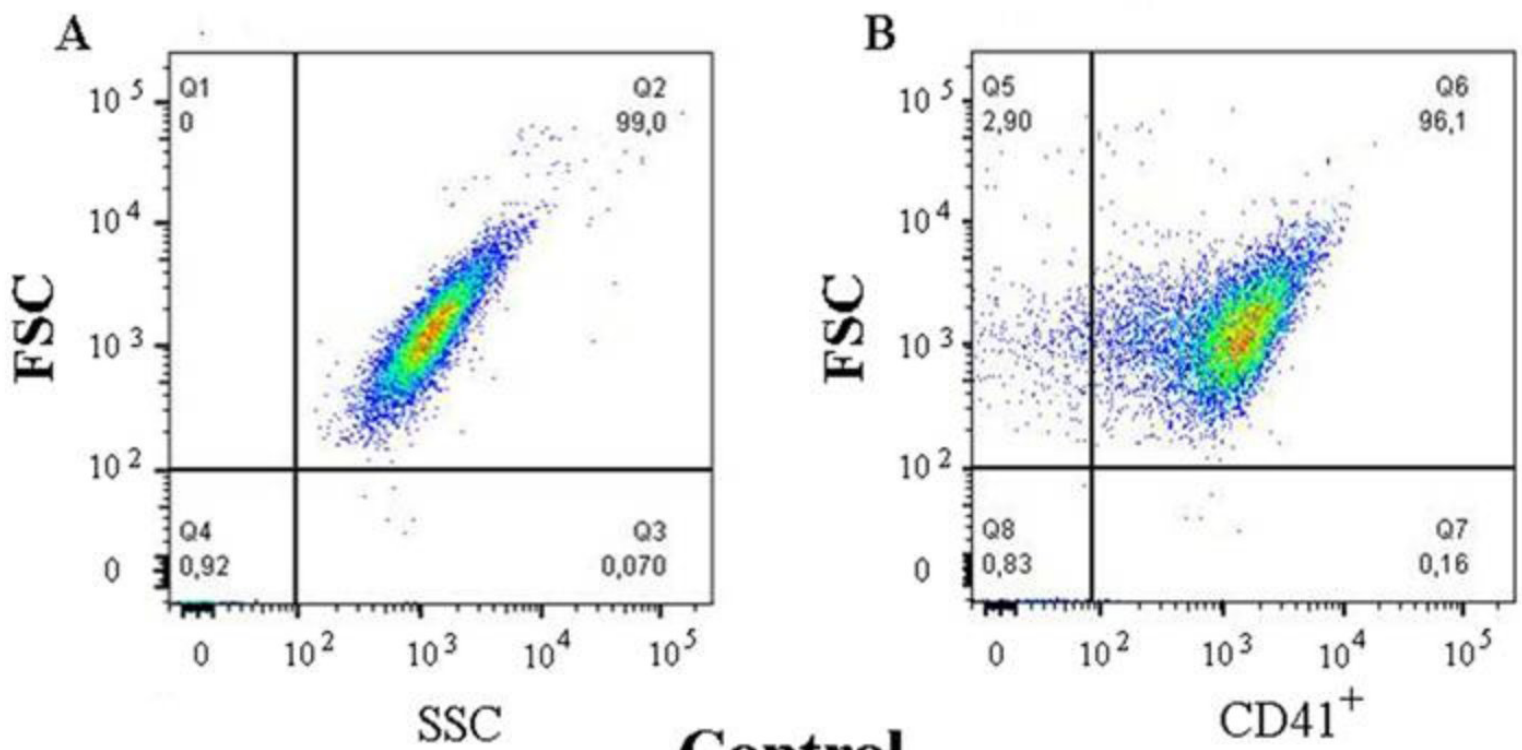

\section{SSC Control}
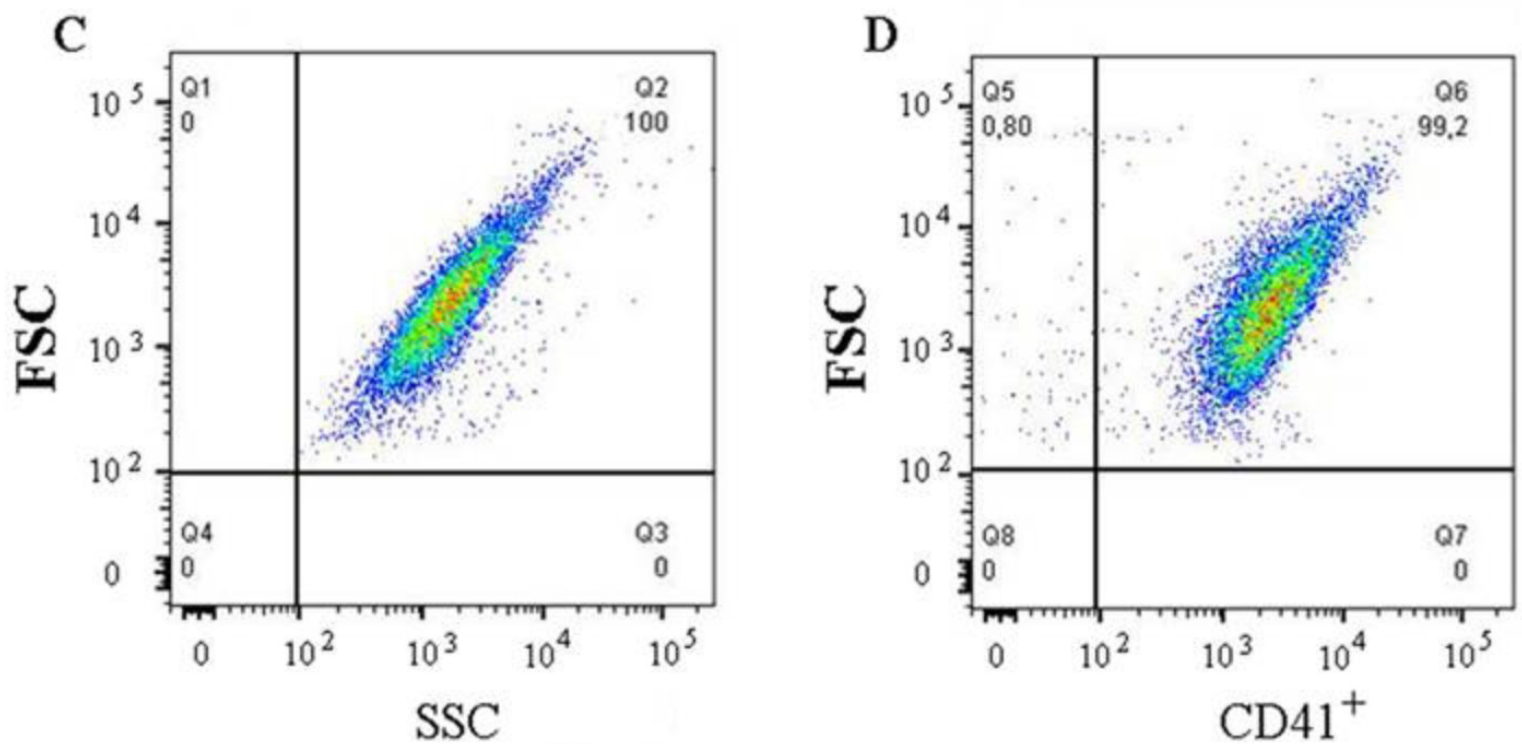

Figure 2. Flow cytometric analysis of CD41 expression in PRP. Representative FSC vs SSC and FSC vs CD41 flow cytometry plots. (A and B) PMM2-CDG patient. (C and D) Healthy control. 


\section{Control}

Patient

$\mathbf{A}$

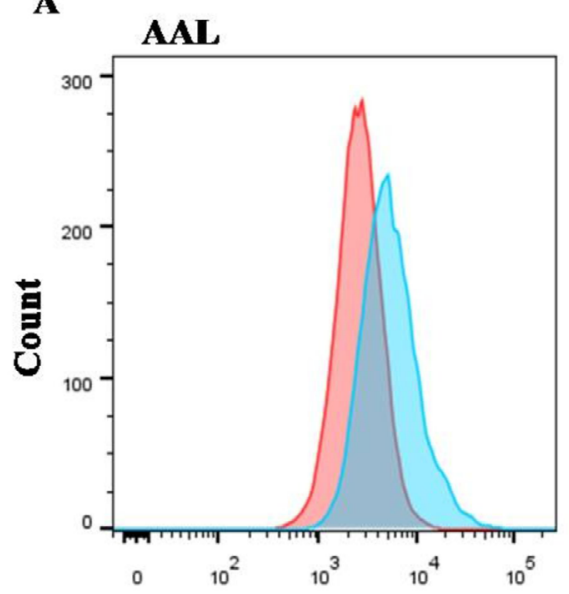

Alexa-647

D

MAA-I

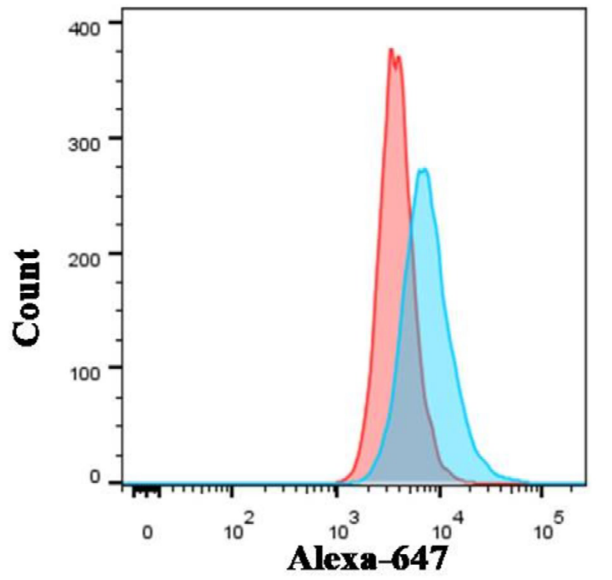

B

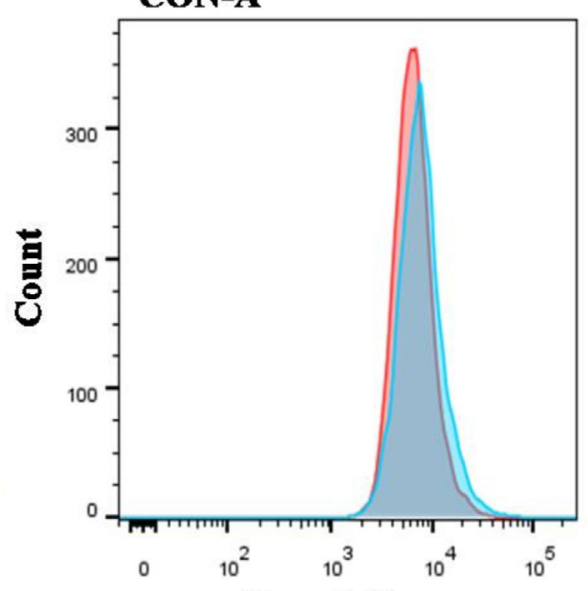

Alexa-647
C

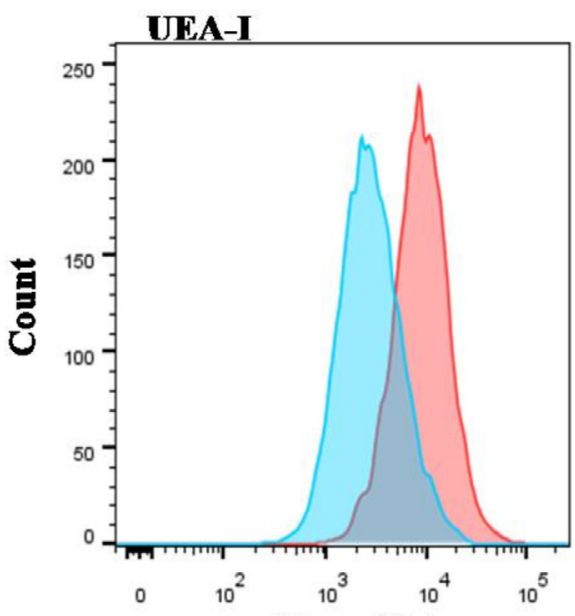

Alexa-647

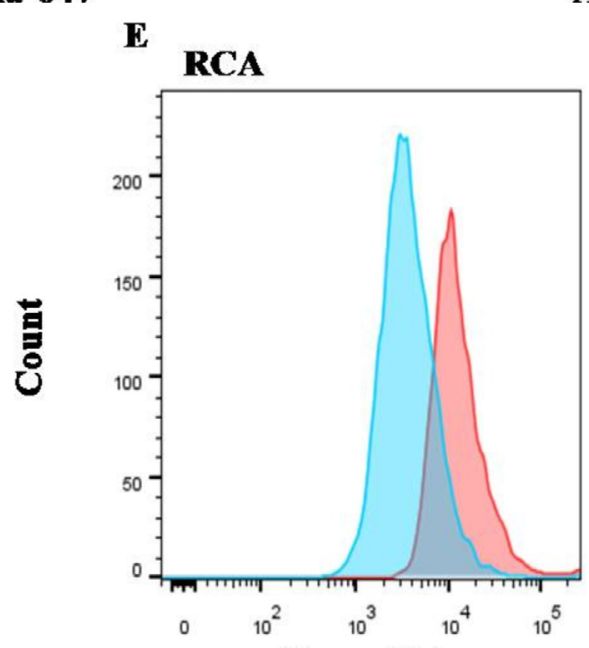

Alexa-647

\begin{tabular}{|c|c|c|c|c|c|c|c|}
\hline \multirow{2}{*}{ Samples } & \multicolumn{5}{|c|}{ MFI Lectins } & \multirow{2}{*}{$\begin{array}{l}\text { Alexa- } 647^{+} \\
\text {cells }\end{array}$} & \multirow{2}{*}{$\begin{array}{l}\text { Statistical } \\
\text { Analysis }\end{array}$} \\
\hline & AAL & CON-A & MAA-I & $\underset{\text { I }}{\text { UEA- }}$ & RCA-I & & \\
\hline Control 1 & 8095 & 12614 & 6875 & 8471 & 5875 & $100 \%$ & \multirow{6}{*}{ NS } \\
\hline Control 2 & 17908 & 26581 & 14837 & 606 & 21281 & $100 \%$ & \\
\hline Control 3 & 5581 & 8399 & 7354 & 3370 & 3616,3 & $100 \%$ & \\
\hline Control 4 & 14306 & 30251 & 9822 & 8965 & 11059 & $100 \%$ & \\
\hline Control 5 & 7946 & 18785 & 6119 & 2563 & 14307 & $100 \%$ & \\
\hline Control 6 & 6873 & 6832 & 5309 & 16220 & 8113 & $100 \%$ & \\
\hline $\begin{array}{l}\text { PMM2- } \\
\text { CDG }\end{array}$ & 3078 & 7102 & 7068 & 8710 & 11585 & $100 \%$ & NS \\
\hline
\end{tabular}

Figure 3. Flow cytometric analysis of platelet surface membrane glycosylation.

Platelet populations were gated based on positive CD41-PE staining and lectins detected by Alexa Fluor 647-conjugated streptavidin. No significant (NS) binding differences were detected between control and PMM2-CDG patient samples for the following 5 lectins: A. AAL (specific for a1,6linked fucose). B. CON-A (specific for a-mannose). C. UEA-I (specific for a-fucose). D. MAA-I (specific for sialic acid bound to galactose a-2,3). E. RCA-I (specific for $\beta$-galactose). Histograms superpose the control (blue peaks) and patient (read peaks) for comparison. F. Summary results. MFI and CD41 ${ }^{+} /$Lectin $^{+}$platelet counts (\%) for each lectin are indicated for control and PMM2-CDG patient samples. 

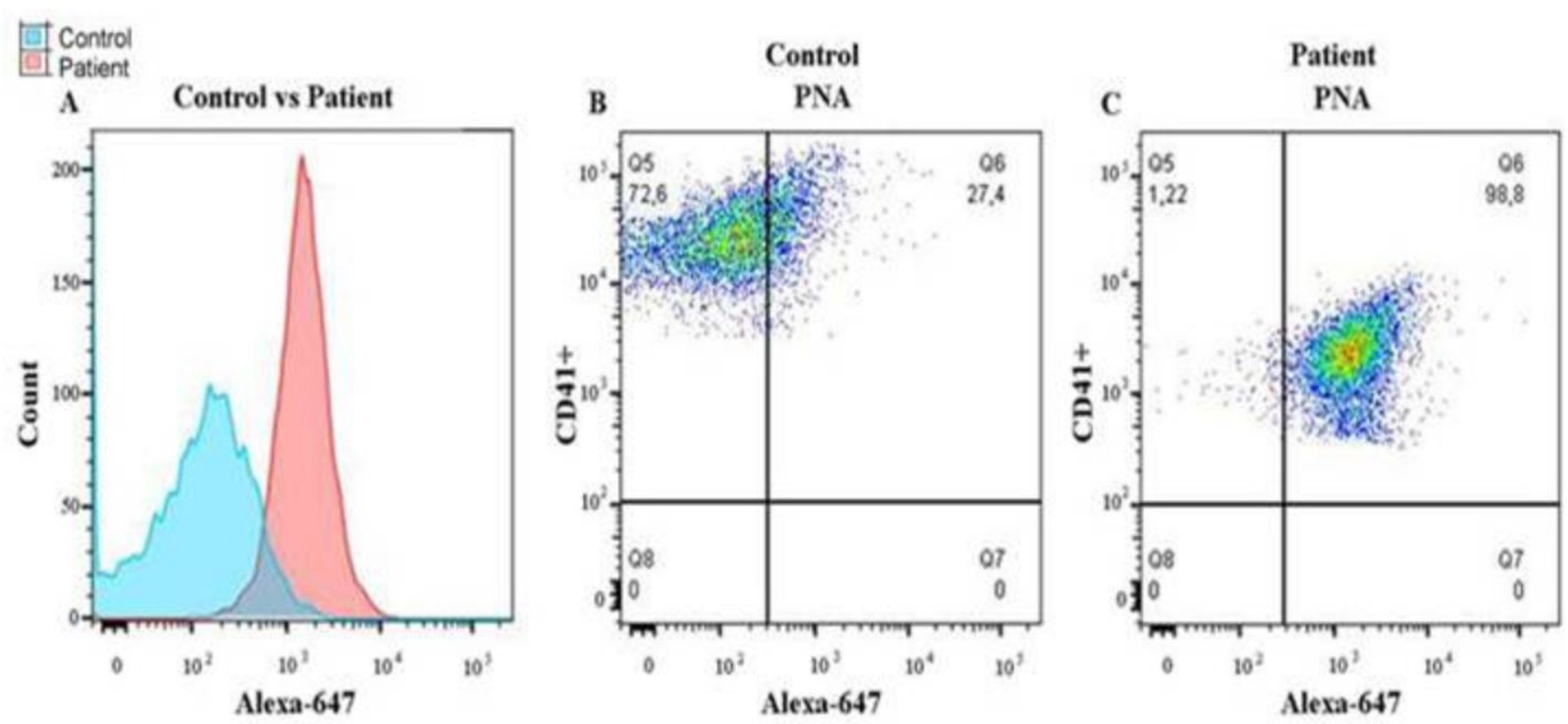

D

\begin{tabular}{|c|c|c|c|}
\hline Samples & $\begin{array}{c}\text { MAPNA } \\
\text { Lectin }\end{array}$ & $\begin{array}{c}\text { Aleva-647* } \\
\text { cells }\end{array}$ & $\begin{array}{l}\text { Statistical } \\
\text { Analysis }\end{array}$ \\
\hline Control 1 & 435 & $51 \%$ & \multirow{6}{*}{ NS } \\
\hline Control 2 & 414 & $44 \%$ & \\
\hline Control 3 & 143 & $27.4 \%$ & \\
\hline Control 4 & 251 & $27 \%$ & \\
\hline Control 5 & 844 & $30 \%$ & \\
\hline Control 6 & 449 & $24 \%$ & \\
\hline PMM2-CDG & 1228 & $98.8 \%$ & $\begin{array}{l}\text { Significant } \\
\text { (p=00065) }\end{array}$ \\
\hline
\end{tabular}

Figure 4. Enhanced binding of PNA to PMM2-CDG platelets.

Platelet populations were gated based on positive CD41-PE staining and PNA lectin binding detected by Alexa Fluor 647-conjugated streptavidin. A. Representative MFI histogram showing PNA signal distribution in a control and a PMM2-CDG patient PRP sample. B-C. Representative dotplots of CD41 expression vs. PNA lectin signal in control and patient PRP samples. D. Summary results. MFI of the PNA signal and CD41 $\%$ $\mathrm{PNA}^{+}$platelet counts(\%) are indicated for control and PMM2-CDG patient samples. 
Control
Patient

A Control vs Patient

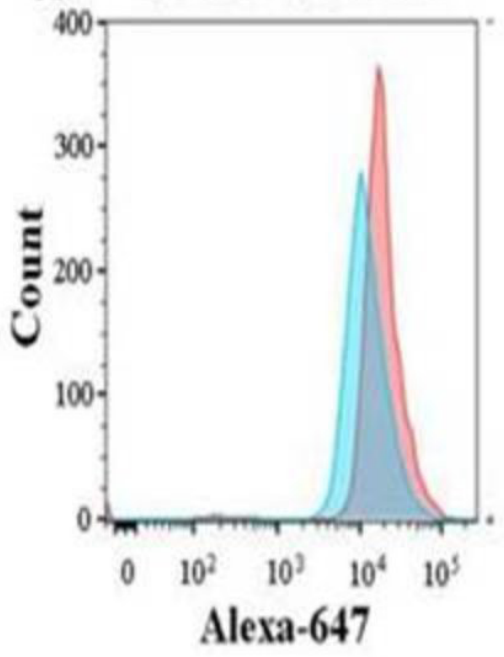

D

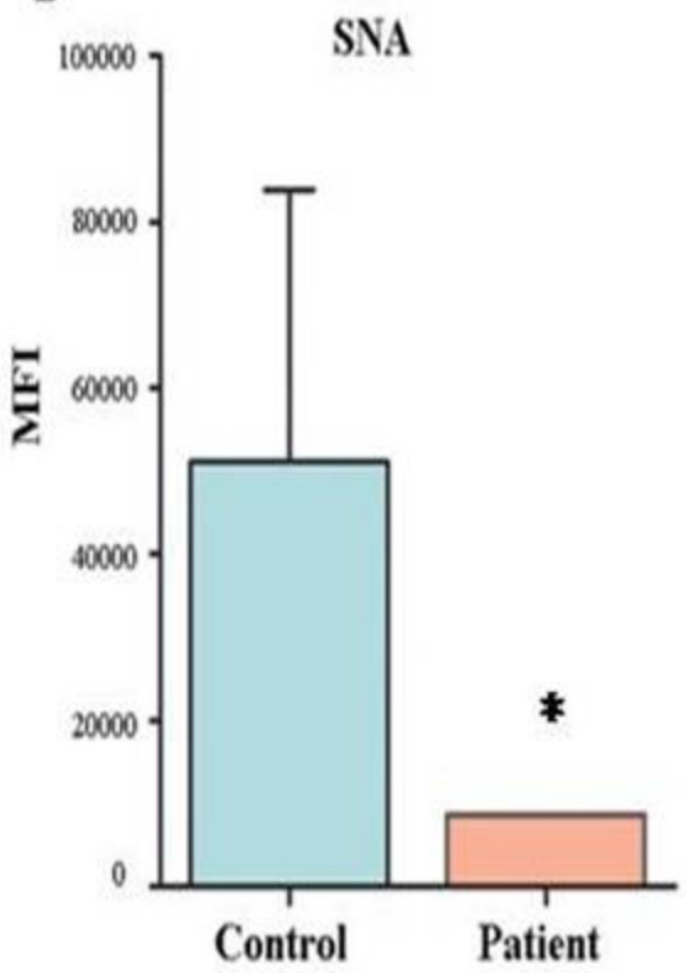

Control

B

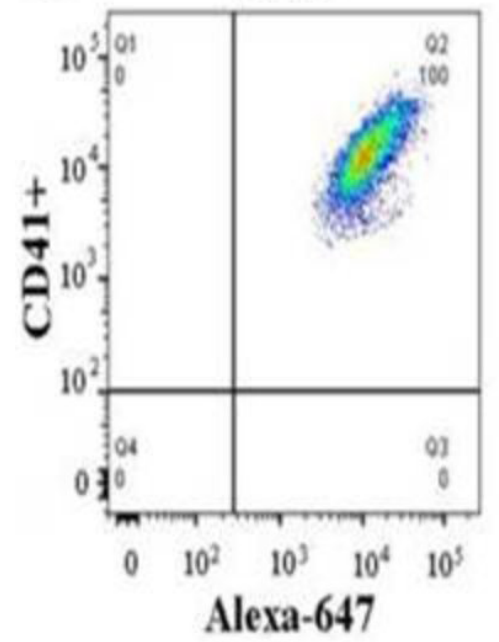

Patient

C SNA

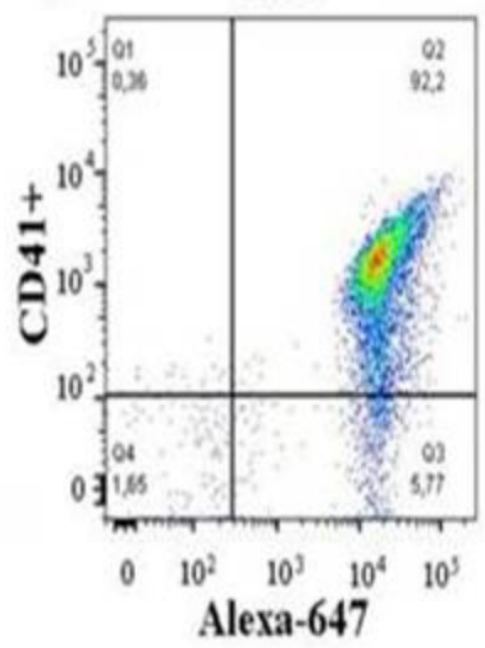

E

\begin{tabular}{|c|c|c|c|}
\hline Samples & $\begin{array}{l}\text { MFISNA } \\
\text { Leclin }\end{array}$ & $\begin{array}{c}\text { Alexa- } \\
647+\text { cell } \\
\text { (\%) }\end{array}$ & $\begin{array}{l}\text { Stalistical } \\
\text { Analysis }\end{array}$ \\
\hline Cootral 1 & 52784 & $100 \%$ & \multirow{6}{*}{ NS } \\
\hline Control 2 & 36261 & $100 \%$ & \\
\hline Control3 & 15910 & $100 \%$ & \\
\hline Contral 4 & 16031 & $100 \%$ & \\
\hline Contral5 & 16293 & $100 \%$ & \\
\hline Condrol 6 & 69776 & $100 \%$ & \\
\hline PAME-CDX & 9341 & $100 \%$ & $\begin{array}{l}\text { Significant } \\
\text { (p=(10238) }\end{array}$ \\
\hline
\end{tabular}

Figure 5. Decreased binding of SNA to PMM2-CDG platelets.

A. Platelet populations were gated based on positive CD41-PE staining and SNA lectin binding detected by Alexa Fluor 647-conjugated streptavidin. Representative MFI histogram showing SNA signal distribution in a control and a PMM2-CDG patient PRP sample. B-C. Representative dot plots of CD41 expression vs. SNA lectin signal in control and patient PRP samples. D. SNA-MFI summary data (* =0.0238). E. Summary results. MFI for SNA signal and CD41+/SNA ${ }^{+}$platelet counts (\%) are indicated for control and PMM2-CDG patient samples. 


\section{Discussion}

This study provides the first account of a glycophenotypic characterization of platelet membrane proteins in a PMM2-CDG patient. Although the PMM2-CDG patient reported here did not present overt bleeding events, the analysis of platelet aggregation showed a decreased response to ADP and epinephrine, revealing a potential susceptibility to haemostatic complications during traumatic injury or surgery. In most individuals, a biphasic aggregation response is observed with low concentrations of ADP and epinephrine, while unimodal aggregation curves are typically generated by other agonists (e.g., collagen and ristocetin) [12]. In this patient, we also detected low levels of Factor IX and XI, KPTT at the normal upper limit (which could be associated with the decrease in Factor IX), and normal values for coagulation inhibitors.

Although alterations in glycosylation are the defining characteristic of CDG, some gaps remain in our understanding of their actual contribution to haemostatic alterations. In particular, for many CDG types the glycan profile of platelet membranes has yet to be defined. Lectins represent a useful tool for recognizing specific carbohydrates and identifying changes in glycosylation patterns on the surface of the cells. We used a panel of 7 lectins to describe the glycosylation profile of surface proteins in platelet membranes. Compared to control samples, platelets from the PMM2-CDG patient showed both increased binding intensity (higher MFI) and a higher positivity rate for PNA, which recognizes $\beta$-1- $N$-acetylgalactosamine sequences and some non-sialylated structures, including some O-glycoproteins. In turn, significantly decreased binding of SNA lectin, which recognizes terminal $\alpha-2,6$-linked sialic acids in glycoproteins, but no difference in platelet SNA positivity rate, were detected in patient's vs controls' samples. No significant differences in MFI nor in the percentage of positive platelets were observed between patient's and controls' samples for the remaining 5 lectins, namely AAL, CON-A, UEA-I, RCA-I, and MAA-I. Since PNA shows binding specificity for terminal galactose, higher PNA expression in platelet surface proteins from the PMM2-CDG patient appears to indicate higher abundance of desialylated core $1 O$-glycans. Although these distinct PNA and SNA binding profiles suggest defective sialylation in PMM2CDG platelets, further PMM2-CDG cases are to be evaluated to validate these findings.

Sialylated $\mathrm{N}$-glycans in mammalian serum proteins have different linkage types $(\alpha 2,3$ and $\alpha 2,6)$ and contain one to three sialic acid molecules (mono-, di-, and tri-sialyl). It is unclear, however, how these alternative conformations impact on $\mathrm{N}$-glycans functions $[18,19]$. The composition of terminal sialic acid residues in $\mathrm{N}$-glycans was shown to modulate the activities of numerous circulating glycoproteins by affecting their stability, enzymatic activity, serum half-life, and organ specificity [19-21]. Differences in $N$-glycan structures (including sialyl Le ${ }^{a / x}$ epitopes and di-sialyl antennae), $\mathrm{N}$-glycosylation occupancy, as well as the length of the $\mathrm{N}$ terminus, may all explain the large heterogeneity observed in the size of human blood-derived Protein C inhibitor (PCI) [22]. Similarly, modifications in the N-glycan profile of platelet surfaces may potentially lead to an altered interaction with other platelets or the blood vessel wall, resulting in the disruption of normal homeostatic mechanisms that regulate platelet aggregation [23].

Although many platelet glycoproteins are predominately modified by O-glycans [24], the biological importance of O-glycans in platelet homeostasis remains unclear. King et al. (2017) [25] used a dual Vicia Villosa Agglutinin (VVA) and Peanut Agglutinin (PNA) lectin binding assay to conduct the first large-scale identification of O-glycosites in platelets, plasma, and endothelial cells [25]. Meanwhile, Li et al. (2018) demonstrated a key role of posttranslational O-glycosylation in platelet homeostasis by showing that platelets lacking O-glycans have a reduced lifespan and are subject to increased clearance in the liver due to defective sialylation. These authors further showed that hepatic macrophages (Kupffer cells) play a major role in clearing desialylated O-glycan-deficient platelets in cooperation with hepatocytes via the hepatic asialoglycoprotein receptor [26].

The hyposialylated profile of PMM2-CDG platelets is arguably a consequence of the defective glycosylation machinery in these patients, and likely impairs the normal function of platelet surface glycoproteins. This might thus explain the spontaneous platelet reactivity described in PMM2-CDG patients [27,28]. In addition, decreased sialic acid expression could increase platelet clearance and lead to thrombocytopenia in peripheral blood, increasing the risk of bleeding in these patients [15].

In conclusion, this work contributes to the knowledge of the physiopathology of systemic hypoglycosylation in CDG by describing the glycophenotype of platelet membrane proteins in a PMM2-CDG patient. We detected a predominance of $\mathrm{N}$-glycosylated surface proteins and significantly diminished terminal sialic acid contents in our patient's PRP samples, which would likely stimulate platelet clearance leading to thrombocytopenia and an increased risk of hemorrhage. The reactivity of such abnormally glycosylated proteins might also account for the platelet hypo-responsiveness to agonists described in these patients. Nevertheless, since only one PMM2-CDG patient was included in this study, a larger sampling is needed to generalize our findings. Further studies evaluating the functional consequences of platelet glycosylation and sialylation defects will help to better understand the physiopathogenesis of PMM2-CDG and hopefully will guide therapeutic strategies to improve life quality in affected patients. 


\section{Acknowledgments}

We are grateful to the Argentinean physicians and biochemists that collaborated toward a better understanding of CDG in Latin American countries. We dedicate this article to the CDG patients and families.

\section{Funding}

This research received grants from CONICET 2017-2021 (RD: 2191), FONCyT PICT 2824; Catholic University of Córdoba 2015-2019, and the Health Ministry of Córdoba, Argentina.

\section{Ethics Approval and Consent to Participate}

The procedures used in this research project were reviewed and approved by the Ethics Committee of the Children's Hospital of Córdoba (CIEIS) Act No 95/2005/2007/2016. The study was performed in accordance with the ethical standards stated in the 1964 Declaration of Helsinki and its later amendments. Written informed consent to participate in this study was obtained from patients and/or their parents.

\section{Declaration of Conflicting Interests}

The authors declare that they have no conflicts of interest to report regarding this manuscript.

\section{References}

1. Ng BG, Freeze HH. Perspectives on glycosylation and its congenital disorders. Trends Genet. 2018;34(6):466-476. doi: 10.1016/j.tig.2018.03.002

2. Jaeken J, Péanne R. What is new in CDG? J Inherit Metab Dis. 2017;40(4):569-586. doi: 10.1007/s10545-017-0050-6

3. Linssen M, Mohamed M, Wevers RA, Lefeber DJ, Morava E. Thrombotic complications in patients with PMM2-CDG. Mol Genet Metab. 2013;109(1):107-111. doi: 10.1016/j. ymgme.2013.02.006

4. Jaeken J. Congenital disorders of glycosylation. Ann N Y Acad Sci. 2010;1214:190-198. doi: 10.1111/j.1749-6632. 2010.05840.x

5. Altassan R, Péanne R, Jaeken J, et al. International clinical guidelines for the management of phosphomannomutase 2-congenital disorders of glycosylation: diagnosis, treatment and follow up. J Inherit Metab Dis. 2019;42(1):5-28. doi: 10.1002/jimd.12024

6. Asteggiano CG, Papazoglu M, Millón MBB, et al. Ten years of screening for congenital disorders of glycosylation in Argentina: case studies and pitfalls. Pediatr Res. 2018 Dec;84(6):837-841. doi: 10.1038/s41390-018-0206-6
7. Briones P, Vilaseca MA, Schollen E, et al. Biochemical and molecular studies in 26 Spanish patients with congenital disorder of glycosylation type Ia. J Inherit Metab Dis. 2002 Dec;25(8):635-646. doi: 10.1023/a:1022825113506

8. Noelle V, Knuepfer M, Pulzer F, et al. Unusual presentation of congenital disorder of glycosylation type 1a: congenital persistent thrombocytopenia, hypertrophic cardiomyopathy and hydrops-like aspect due to marked peripheral oedema. Eur J Pediatr. 2005;164(4):223-226. doi: 10.1007/s00431004-1611-x

9. de la Morena-Barrio ME, Di Michele M, Lozano ML, et al. Proteomic analysis of platelet N-glycoproteins in PMM2CDG patients. Thromb Res. 2014;133(3):412-417. doi: 10. 1016/j.thromres.2013.12.024

10. Schauer R. Sialic acids as regulators of molecular and cellular interactions. Curr Opin Struct Biol. 2009;19(5):507514. doi: 10.1016/j.sbi.2009.06.003

11. Pluma VHC, Viveros PV, Vega C, Quintero M, Monroy R. Agregometría plaquetaria: el estudio de la agregación de las plaquetas y la disfunción plaquetaria. Med Int Mex. 2011;27(1):58-74.

12. Koltai K, Kesmarky G, Feher G, Tibold A, Toth K. Platelet aggregometry testing: molecular mechanisms, techniques and clinical implications. Int J Mol Sci. 2017;18(8):1803. doi: $10.3390 / \mathrm{ijms} 18081803$

13. Tsuda H, Hattori S, Tanabe S, et al. Screening for aetiology zof thrombophilia: a high prevalence of protein $\mathrm{S}$ abnormality. Ann Clin Biochem. 1999;36(4):423-432. doi: $10.1177 / 000456329903600404$

14. Smock K, Moser K. What have we learned from coagulation laboratory participation in external quality programs? Int J Lab Hematol . 2019; 1:49-55. doi: 10.1111/ ijlh.12998.

15. Loeliger E. Standardization of diagnostic materials. Laboratory reagents and coagulation assay procedures. Bull World Health Organ. 1973; 48(6):727-36.

16. Li L, Qu C, Wu X, et al. Patterns and levels of platelet glycosylation in patients with coronary heart disease and type 2 diabetes mellitus. J Thromb Thrombolysis. 2018; 45(1):56-65. doi: 10.1007/s11239-017-1573-2

17. Mycroft RH, Mitchell DC, Kay J. An evaluation of statistical procedures for comparing an individual's performance with that of a group of controls. Cogn Neuropsychol. 2002; 19(4):291-299. doi: 10.1080/02643290143000150

18. Ranstam J. Repeated measurements, bilateral observations and pseudoreplicates, why does it matter? Osteoarthritis Cartilage. 2012;20(6):473-475. doi: 10.1016/j.joca.2012. 02.011 
19. Dugan AS, Eash S, Atwood WJ. An N-Linked glycoprotein with alpha(2,3)-linked sialic acid is a receptor for BK virus. J Virol. 2005;79(22):14442-14445. doi: 10.1128/JVI.79. 22.14442-14445.2005

20. Suganuma M, Nomura T, Higa Y, et al. N-glycan sialylation in a silkworm-baculovirus expression system. J Biosci Bioeng. 2018;126(1):9-14. doi: 10.1016/j.jbiosc.2018.01.007

21. Morell AG, Gregoriadis G, Scheinberg IH, Hickman J, Ashwell G. The role of sialic acid in determining the survival of glycoproteins in the circulation. J Biol Chem. 1971;246(5):1461-1467. doi: 10.1016/s0021-9258(19)7 6994-4

22. Broccolini A, Gidaro T, De Cristofaro R, et al. Hyposialylation of neprilysin possibly affects its expression and enzymatic activity in hereditary inclusion-body myopathy muscle. $J$ Neurochem. 2008;105(3):971-981. doi: 10.1111/j.14714159.2007.05208.x

23. Sun W, Parry S, Panico M, et al. N-glycans and the $\mathrm{N}$ terminus of protein $\mathrm{C}$ inhibitor affect the cofactor-enhanced rates of thrombin inhibition. J Biol Chem. 2008;283(27): 18601-18611. doi: 10.1074/jbc.M800608200
24. Mercado CP, Quintero MV, Li Y, et al. A serotonin-induced $\mathrm{N}$-glycan switch regulates platelet aggregation. Sci Rep. 2013;3:2795. doi: 10.1038/srep02795

25. Lopez JA, Chung DW, Fujikawa K, Hagen FS, Papayannopoulou T, Roth GJ. Cloning of the a chain of human platelet glycoprotein Ib: a transmembrane protein with homology to leucine-rich a2-glycoprotein. Proc Natl Acad Sci U S A. 1987;84(16):5615-5619. doi: 10.1073/pnas. 84.16.5615

26. King SL, Joshi HJ, Schjoldager KT, et al. Characterizing the $\mathrm{O}$-glycosylation landscape of human plasma, platelets, and endothelial cells. Blood Adv. 2017;1(7):429-442. doi: 10.1182/bloodadvances.2016002121

27. Li Y, Fu J, Ling Y, et al. Sialylation on O-glycans protects platelets from clearance by liver Kupffer cells. Proc Natl Acad Sci U S A. 2017;114(31):8360-8365. doi: 10.1073/ pnas. 1707662114

28. Van Geet C, Jaeken J, Freson K, et al. Congenital disorders of glycosylation type Ia and IIa are associated with different primary haemostatic complications. J Inherit Metab Dis. 2001;24(4):477-492. doi: 10.1023/a:1010581613821 